\title{
Use of Scanned Detection in Optical Position Encoders
}

\author{
Eric M. Yeatman, Member, IEEE, Paul J. Kushner, and David A. Roberts
}

\begin{abstract}
The use of scanning detection in optical noncontact position sensing is proposed, and a prototype is implemented, using optical scanning of pseudorandom binary sequences printed in bar-code format. Light from a vibrating fiber tip is imaged onto the printed code using a GRIN lens, and the reflected light detected via the same lens, resulting in a single scanner module. Improved flexibility and signal processing possibilities are obtained compared to conventional diode array optical code readers. Performance limitations of scanning position encoders are analyzed and discussed. Suggestions for implementation using microoptoelectromechanical systems are presented.
\end{abstract}

Index Terms-Bar code, encoder, microoptoelectromechanical systems (MOEMS), scanner.

\section{INTRODUCTION}

$\mathbf{T}$ HE measurement of position for rotating and linearly translating parts is a key requirement in automation, and there are a number of well-established approaches to this problem. Of these, optical techniques have a dominant position, owing to their high resolution capability and low cost [1]. Typically, optical encoders make use of a configuration as shown in Fig. 1(a), with a transmissive pattern illuminated from one side, and an imaging lens or lenses and photodiode array on the other. These methods can be used for both absolute and relative measurement, using aperiodic and periodic patterns respectively. A variety of methods based on diffraction from periodic gratings can be exploited to give sub-wavelength resolution, but the measured position is ambiguous owing to the periodicity of the signal. This ambiguity can be overcome by initially zeroing the system, and then counting the number of grating periods passed, but this approach has two disadvantages. The location information is lost if the signal is interrupted, or system memory lost, even briefly, and additional information is usually required to know the direction of movement, in order to track position. Both these restrictions can be overcome by adding additional patterns. A quadrature approach can be used for determining direction, by adding an additional periodic pattern and associated optics shifted in position by a quarter cycle, and comparing the two signals. Meanwhile an aperiodic pattern can provide absolute position information, and while the resolution of aperiodic encoders is usually limited, systems

Manuscript received March 1, 2003; revised August 15, 2003. This work was supported by the U.K. Engineering and Physical Sciences Research Council (EPSRC).

The authors are with the Optical and Semiconductor Devices Group, Department of Electrical and Electronic Engineering, Imperial College, London, U.K (e-mail: e.yeatman@imperial.ac.uk).

Digital Object Identifier 10.1109/TIM.2003.821502 combining the two methods can provide both absolute position and high resolution.

The optical encoders reported to date are all staring systems, in that the signal is collected using a stationary optical system. A disadvantage of such staring systems is their geometric inflexibility. The photodiode arrays are effectively sampling the signal, with the number of samples and their relative spacing fixed by the array; only the sampling period can be altered, by changing the magnification, and even this cannot be done in the near field case. Also, any optical or electrical filtering of the detected signal has to be carried out at each detector element. It would be beneficial for many applications to further miniaturize the optical system, to allow a greater (as well as more flexible) spacing of the code and the detector element, and to collect optical signals from a large number of effective locations without replicating the optics and electronics a great number of times. As a possible way to achieve these benefits, scanned optical position encoding is introduced here for the first time. Scanning the code in the time domain allows the sample positions to be dynamically assigned, and greatly increased in number, and can also permit the use of a variety of signal processing techniques to extract the code under poor signal-to-noise ratios (SNRs).

The optical system we propose uses a resonant scanner with a reflective code, and is illustrated in Fig. 1(b). Here the light from a laser diode or LED is coupled to a fiber or integrated waveguide, the end of which is vibrated using an actuating element. The light from the vibrating tip is focused by a lens onto a printed pattern as a scan line, and the reflected light detected and analyzed. This architecture will lend itself to a single chip solution, where the single chip can contain the whole optical system as well as processing electronics, with connections only for power and the digital position output, the printed code itself being the only external component. Microoptoelectromechanical systems (MOEMS) are a rapidly developing field [2], and microengineered scanners have been demonstrated by a number of researchers [3], [4]. The application illustrated here is well suited to such technologies. Below we present the analysis of the potential performance of scanned optical encoders and their system requirements and present a first implementation of such a system.

\section{ANALYSIS}

\section{A. Absolute Position Encoding}

As stated above, many optical encoders combine a high resolution periodic code with an aperiodic code to give absolute position [5], [6], the latter requiring a resolution only on the order 


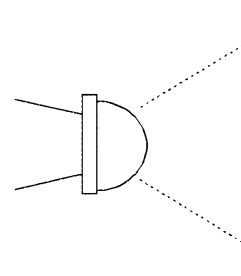

(i)

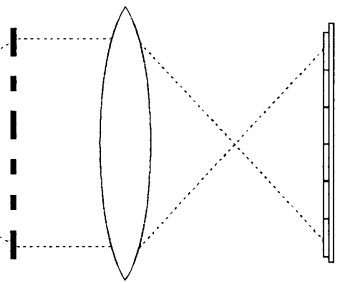

(iii) (iv)

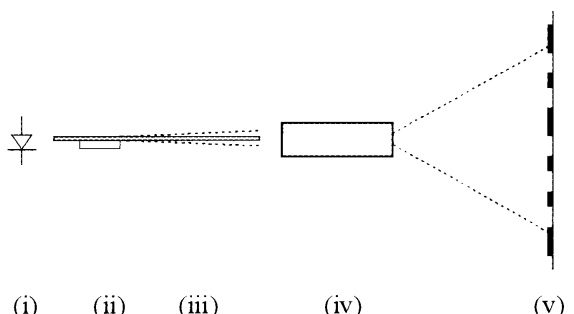

(i) (ii) (iii) (iv) (v)

(a)

(b)

Fig. 1. (a) Schematic of conventional encoder: (i) light source; (ii) transmissive code; (iii) lens; (iv) detector array. (b) Schematic of scanned fiber bar code reader: (i) light source; (ii) actuator and detector elements; (iii) vibrating fiber or waveguide; (iv) lens; (v) reflective code.

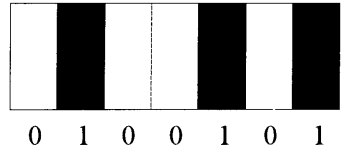

(a)

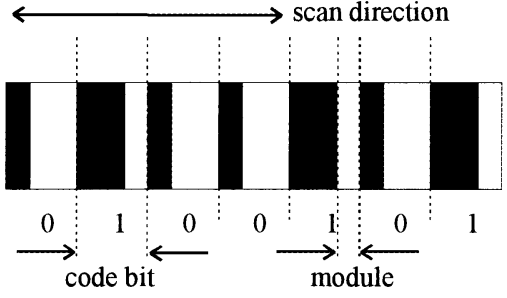

(b)

Fig. 2. (a) Conventional format of NRZ position code; (b) format of $(n, k)=(3,1)$ scanned position code.

of the periodicity of the former. Typically, a discrete sequence of codes of length $n$ bits is used, giving a total measurement range $2^{n}$ times the code segment size. While laterally written codes (code sequence perpendicular to the direction of travel) at each location can be used, overlapping codes written in the direction of translation will be far more space efficient. Good [7] showed how suitable code sequences can be constructed based on graph theory, and Bondy and Murty suggested their use in absolute rotational position encoding on a drum [8]. The codes, or pseudorandom binary sequences (PRBS), are formed such that for a code length of $\left(2^{n}-1\right)$ bits, no two segments of length $n$ are alike, giving one measurable position per code bit. Shift register sequences provide a convenient implementation of the formation of such codes, and the feedback logic required to form maximal length shift register sequences for a given value $n$ are well known [9]. The use of PRBS for absolute position encoders, based on electrical sensing of a line array of insulating and conducting segments, is long established [10].

The maximal length sequence technique also lends itself to optical implementation. The code can be written as a series of transparent and opaque slots, lit from behind, and detected by an array of photodiodes either in the near field of the code or combined with an imaging lens [2]. The detection arrangement for conventional PRBS optical position codes usually consists of a photodetector array with one element per code bit, the code being written in simple nonreturn-to-zero (NRZ) format as illustrated in Fig. 2(a). To digitize the data without loss of information, we need only sample at the Nyquist limit, i.e., $2 \Delta \mathrm{f}_{s}$, where $\Delta \mathrm{f}_{s}$ is the maximum spatial frequency of the signal. Since NRZ data can be reasonably recovered with a bandwidth half the bit-rate, this sets the Nyquist limit as one sample per bit, which is of course exactly the number used in the staring array. The band-limiting filtering is carried out by the detector array itself, effectively performing a piecewise average by integrating the light on each element. The sampling position is determined by the relative position of the array and the code, leading to increasingly strong inter-symbol interference (ISI) as the two become misaligned. To reduce this problem, over-sampling can be employed [3], or a second code can be provided, with additional detectors, for synchronization [11]. In the latter case, memory is employed to track direction, and hence part of the advantage of absolute encoding is lost.

If the code is continuously read by a scanner, however, the ISI problem above can be eliminated. The sampling rate is now limited only by the speed of the detector electronics, and the transitions can be found dynamically. In some senses, the signal obtained is similar to that of an optical data transmission system, and thus we can use similar analysis to determine required signal levels. The key difference is in clock recovery. The scanned signal will consist of a series of sequences (the individual scan lines) which are not mutually synchronised, and thus the clock must be obtained from each sequence of code length $n$, where $n$ is likely to be $<20$. Furthermore, the regular spatial periodicity of the physical code will be distorted unless the scan line moves across it with constant velocity, which is unlikely.

Fortunately, this problem has been extensively studied in the context of bar code reading, where, again, short binary spatial sequences are scanned optically. Clock recovery is typically done by edge detection, and bar codes also share the property of being read in reflection rather than transmission. Unlike conventional position codes, bar codes are not written as arbitrary binary NRZ sequences, but as code words with restricted properties, in order to ensure a sufficient number of transitions for clock recovery as well as to add redundancy for error correction. Most bar-code words consist of a series of modules of equal length, each colored white (spaces) or dark (bars). In the so-called delta codes, a code family $(n, k)$ has words of length $n$ modules, within which there are k pairs of bars and spaces (the bars and spaces each being an integral number of modules wide) [12]. Of these, the information content is maximized for 


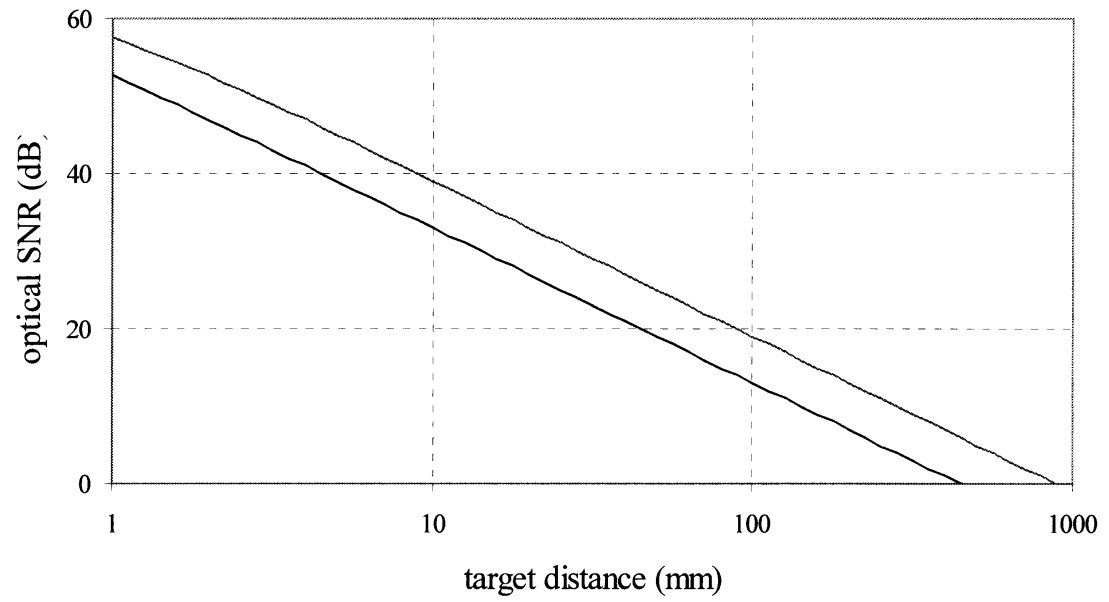

Fig. 3. SNR for scanned position encoder versus target distance, for pupil diameters of the detector optics of $1 \mathrm{~mm}$ (lower line) and $2 \mathrm{~mm}$.

symmetric codes having $n=4 k-1$. The information content in bits per module increases, and approaches 1 , with increasing $\mathrm{k}$, as the number of possible code words approaches $2^{n}$. Width codes consist of alternating bars and spaces, with the width of these being varied, so that an $(\mathrm{m}, \mathrm{w})$ code family has m elements (bars and spaces) of which $\mathrm{w}$ are wide. Here, the information content increases with w.

In our application of PRBS position codes, we have the additional restriction that adjacent code words of length $n$ must overlap by $(n-1)$ bits. This is only a straightforward requirement if the code words have a bit-by-bit correspondence to, for example, a maximal length shift register sequence. We, therefore, chose the simplest possible symmetric $(n, k)$ code, i.e., $(n, k)=(3,1)$, such that each code bit (or symbol) is written as a bar-space pair of total length 3 modules, with spaces of one or two modules in length corresponding to 1 or 0 in the original code. This is illustrated in Fig. 2(b). The information content is low at $1 / 3$ bits per module, but the two transitions per bit greatly ease clock, and thus data, recovery.

\section{B. Signal-to-Noise Ratio}

Let us consider the SNR of the scanning system. If light from a waveguide end is imaged onto the bar code using a lens of greater numerical aperture (NA) than the waveguide, little light will be lost on transmission. If we assume that the reflection from the illuminated surface is Lambertian [13], then the fraction of transmitted light received by the detector can then be approximated by $\sin ^{2}(\mathrm{D} / 2 d) R$, where $R$ is the reflectivity of the bar code, $\mathrm{d}$ is the distance to the target and $\mathrm{D}$ the diameter of the detector optical system (assuming $d \gg \mathrm{D}$ ). This approximation also assumes that the scanning spot is much smaller than D, and that the maximum scan angle is small; in practice, the finite size of both the scanned spot and the scan angle will reduce the detected power level. The bandwidth $\Delta \mathrm{f}$ needed by the detector is determined by the effective bit-rate $\mathrm{B}$, according to the Nyquist criterion $\Delta \mathrm{f}=\mathrm{B} / 2$, where $\mathrm{B}$ is the inverse of the shortest time taken to scan one code module (i.e., $B$ is the module rate in this case). Let $\mathrm{f}_{s}$ be the scanning frequency, and we introduce a quantity $\nu$ equal to the fraction of the scan time which is used to read the code, the edges of the scan line being discarded to reduce distortion. Then if we also discard the reversed image of the code obtained during the return part of the scan, the time spent scanning the code is $\nu / 2 \mathrm{f}_{s}$. If $n$ is the number of bits in each code word (total code length $2^{n}-1$ ) and we use the encoding technique described above with 3 code modules per bit, then

$$
\mathrm{B}=6 n f_{s} / \nu
$$

and $\Delta \mathrm{f}=3 n f_{s} / \nu$. Electrical (including thermal and amplifier) noise can be taken into account through a noise equivalent power (NEP) of the receiver. If this is the dominant noise source, then we obtain

$$
\mathrm{SNR}=\frac{\sin ^{2}(\mathrm{D} / 2 d) R \Phi_{\mathrm{T}}}{\mathrm{NEP}} \sqrt{\frac{\nu}{3 n f_{s}}}
$$

(defined in terms of the optical power), where $\Phi_{T}$ is the transmitted power. Taking reasonable values of $\Phi_{T}=1 \mathrm{~mW}, \nu=$ $0.8, \mathrm{NEP}=10 \mathrm{pW} / \sqrt{ } \mathrm{Hz}, n=16, R=1$, and $\mathrm{f}_{s}=300 \mathrm{~Hz}$, for example, we get $\mathrm{SNR}=53 \mathrm{~dB}-20 \log (d / \mathrm{D})$. From this we can determine SNR versus target distance $d$ for various pupil sizes of the detection optics, as shown in Fig. 3. In optical communication systems, to achieve a bit error rate better than $10^{-9}$ an optical SNR of about $10 \mathrm{~dB}$ is sufficient, and this implies a possible target distance in our system of greater than $10 \mathrm{~cm}$.

Here, we have included, in the noise equivalent power, only receiver related noise, but there will also be shot noise in the detected signal, the magnitude of which depends on received signal intensity. We can write an expression for NEP for shot noise only as

$$
\mathrm{NEP}_{\mathrm{sh}}=\sqrt{\frac{2 h \mathrm{c}}{\eta \lambda} \Phi_{\mathrm{R}}}
$$

where $\mathrm{h}$ is Planck's constant, $\mathrm{c}$ the speed of light, $\lambda$ the wavelength, $\eta$ the detector quantum efficiency, and $\Phi_{\mathrm{R}}$ is the received optical power. For wavelengths in the near infra-red or visible, we find that $\mathrm{NEP}_{\text {sh }}$ only reaches significant levels (compared to receiver noise) for received optical powers around $1 \mathrm{~mW}$. For example, taking $\eta=1, \lambda=0.7 \mu \mathrm{m}$, and $\Phi_{\mathrm{R}}=1 \mathrm{~mW}$ gives $\mathrm{NEP}_{\text {sh }}=24 \mathrm{pW} / \sqrt{ } \mathrm{Hz}$. For reasonable values of $\Phi_{T}$ and $\mathrm{d} / \mathrm{D}$, $\Phi_{\mathrm{R}}$ will be well below $1 \mathrm{~mW}$ and shot noise can be neglected. 


\section{Ambient Light}

The low effective bandwidth implies very low receiver noise values, and, therefore, ambient light may also be a significant noise source, both through shot noise, and through any modulation of the ambient light that is within the relevant bandwidth of the receiver circuit. However, the relatively low numerical aperture of the receiver in the scanned system greatly reduces the amount of ambient light collected. Gfeller and Pabst [13] have studied the levels of various ambient light sources, and measured the resulting photocurrents, in a $1 \mathrm{~cm}^{2}$ silicon photodiode with field of view (FOV) of $50^{\circ}$, with and without optical filters. They obtain photocurrents as high as a few $\mathrm{mA}$ in the unfiltered case (equivalent to a few $\mathrm{mW}$ received optical power), but for a scanner using cladding modes of an optical fiber for detection as proposed here, these levels will be reduced by over three orders of magnitude. In this case, shot noise will not be a concern.

Modulation on the ambient sources may, however, be significant. Moreira et al. [14] have measured the modulation spectrum of various sources. Solar radiation has only slowly moving components (a few hertz or below) associated with moving objects and the sun's motion across the sky. Incandescent lamps have a strong component at twice the mains frequency (i.e., at 100 or $120 \mathrm{~Hz}$ ), with higher harmonics $40 \mathrm{~dB}$ or more weaker. Most problematically, fluorescent lamps with electronic ballasts produce high spectral densities from $100 \mathrm{~Hz}$ to $100 \mathrm{~s}$ of $\mathrm{kHz}$. Fortunately, the optical spectrum of fluorescent lamps is limited, with negligible power at wavelengths longer than about $0.85 \mu \mathrm{m}^{14}$. To eliminate this source, while still allowing the use of low cost silicon photodiodes, an operating wavelength of about $0.9 \mu \mathrm{m}$ is ideal. In conclusion, a combination of optical and electrical filtering appears able to reduce the effects of ambient light well below those of receiver electrical noise.

Low-pass filtering is standard in detection of optical signals, and by analogy with optical on-off keying modulation, a bandwidth of half the module rate in our case is adequate to maintain signal integrity. Setting a high-pass cutoff is less straightforward, as these are baseband signals. However, the $\mathrm{RZ}$ encoding format we have chosen is more tolerant to high pass filtering than NRZ codes. Choosing a simple first order $R C$ filter with cutoff frequency $f_{o}=1 / 2 \pi R C$, the eye-closing penalty will increase with increasing $f_{o}$, and to keep this penalty below $1 \mathrm{~dB}$ requires approximately $\mathrm{f}_{\mathrm{o}}<\mathrm{B} / 20$. This is illustrated in Fig. 4, for a short position code sequence. The first order filter will give $20 \mathrm{~dB}$ or more attenuation for frequencies below $\mathrm{f}_{\mathrm{o}} / 10$, so to achieve 20 - $\mathrm{dB}$ attenuation at the problematic frequencies of 100 or $120 \mathrm{~Hz}$ requires $\mathrm{f}_{\mathrm{o}}>1 \mathrm{kHz}$. For the previous examples of $\nu=0.8, n=16$, and $\mathrm{f}_{s}=300 \mathrm{~Hz}, \mathrm{~B}$ will be $36 \mathrm{kHz}$, so for $f_{o}=1 \mathrm{kHz}$ we have $f_{o}=B / 36$. This shows low-frequency cutoff to be feasible; and with higher order filtering the effect on the signal could be made negligible. Alternatively, we have the possibility of modulating the optical source with a sub-carrier at well above B, then bandpass filtering and demodulating. Although this will add to the complexity and cost of the analog circuitry, it is much more practical than would be the case with staring encoders, where complete receiver circuits are needed for each of $n$ detectors.

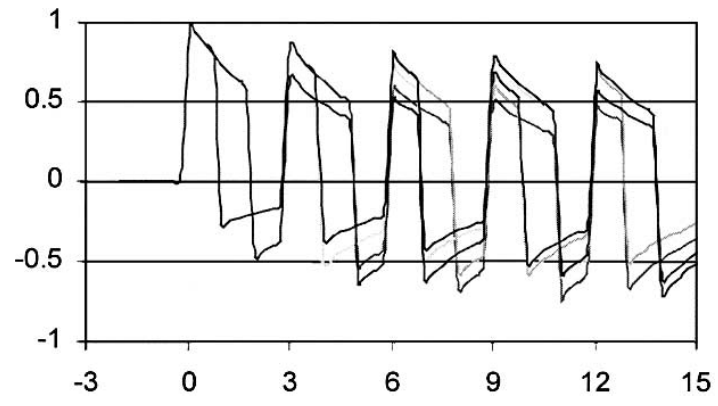

Fig. 4. Eye diagram for first 5 bits of position code sequence, after high pass filtering by a first order filter with cutoff frequency $f_{o}=B / 20$, where $B$ is the module rate.

\section{Spatial Resolution}

Spatial resolution is limited by the optical system for both scanned and staring encoders, particularly where an imaging system is used (as opposed to near field detection, frequently used by staring arrays). In the scanning case, the detected signal is effectively the convolution of the bar code with the image of the scanner spot [15]. This also causes ISI, increasingly as the spot size increases compared to the code element size. In the case of a waveguide scanner [16], as used here, the smallest possible spot size is effectively the mode size of the waveguide, multiplied by the magnification of the optical system. Since the ratio of the scan range to spot size is, to a first order approximation, independent of magnification, the effect of this convolution (blurring) on the data recovery is then a function of $n$, the number of code bits, versus the ratio $\rho_{\mathrm{w}} / \sigma_{\mathrm{w}}$, where $\sigma_{\mathrm{w}}$ is the waveguide output spot radius and $\rho_{\mathrm{w}}$ the maximum waveguide displacement from equilibrium during scanning (the length of the scan line on the bar code will be $2 \rho_{\mathrm{w}} M$, where $M$ is the magnification of the optical system). The ISI due to spot size is similar to that caused by dispersion in digital fiber optic systems [17]; as in such systems, it should be possible to reduce substantially the ISI with a suitably designed filter, such as that introduced by Turin and Boie [18]. However, SNR will still suffer because of the reduced amplitude of the spread code segment at the sampling point. Let us assume the scanning spot has a Gaussian intensity distribution

$$
P(x)=\frac{\Phi_{\mathrm{o}}}{\sqrt{\pi} \sigma_{x}} e^{-x^{2} / \sigma_{x}^{2}}
$$

where $x$ is the scanning direction, such that its total intensity $\Phi_{\mathrm{O}}$ is independent of spot size. For a code module width w, and scan velocity $v_{s}$, the module width and spot radius in the time domain will be $t_{\mathrm{o}}=\mathrm{w} / v_{s}$ and $\sigma_{\mathrm{t}}=\sigma_{x} / v_{s}$ respectively, and the photocurrent signal from a single module will be given by the convolution

$$
\mathrm{I}(t)=C \frac{\Phi_{\mathrm{o}}}{\sqrt{\pi} \sigma_{t}} e^{-t^{2} / \sigma_{t}^{2}} \otimes\left(U\left(t-t_{\circ} / 2\right)-U\left(t+t_{\mathrm{o}} / 2\right)\right)
$$

where $\mathrm{C}$ is a constant including the efficiency and responsivity of the detection system, and $\mathrm{U}(\mathrm{t})$ is the unit step function. Following the analysis of [18], we can show that the peak value of this convolution will be proportional to

$$
\mathrm{I}_{\max } \propto \frac{2}{t_{o}} \operatorname{erf}\left(\frac{t_{o}}{2 \sigma_{t}}\right) .
$$




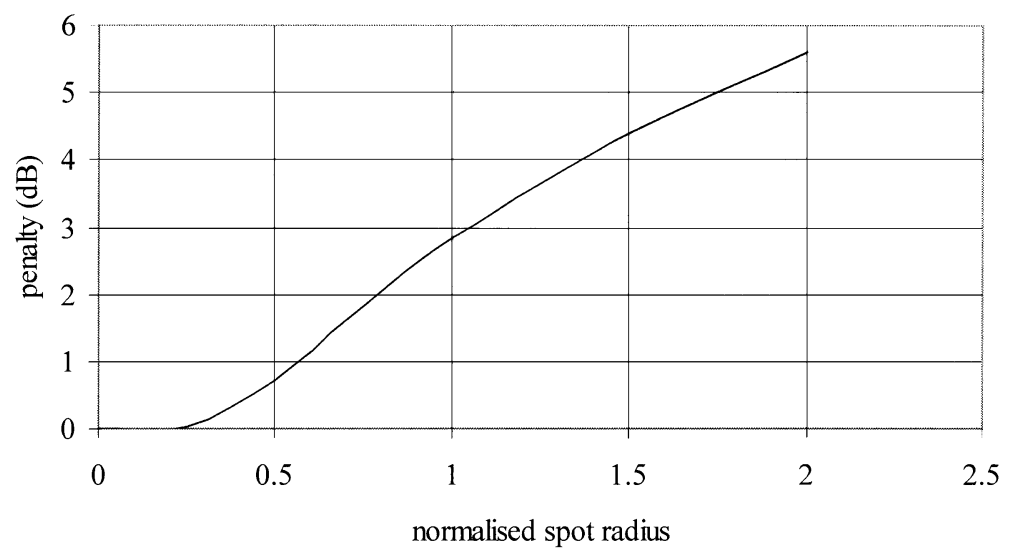

Fig. 5. Power penalty for reduction of peak signal level by blurring versus normalized spot size $\sigma_{x} / \mathrm{w}$.

Note that the useful scan length $2 \nu \rho_{\mathrm{w}} M=3 \mathrm{nw}$, and $\sigma_{x}=$ $\mathrm{M} \sigma_{\mathrm{w}}$, so that the point spread parameter

$$
\frac{t_{\mathrm{o}}}{\sigma_{t}}=\frac{w}{\sigma_{x}}=\frac{2}{3} \nu \frac{\rho_{w}}{n \sigma_{w}} .
$$

We can now use (6) to calculate a SNR penalty as a function of the normalized spot size $\sigma_{x} / \mathrm{w}$, and this is illustrated in Fig. 5. The penalty is only $3 \mathrm{~dB}$ for a spot radius (standard deviation) of $\sigma_{x}=\mathrm{w}$, i.e., for a full-width at half-power spot size just less than the code bit size $3 \mathrm{w}$.

\section{E. Vibration}

Susceptibility to mechanical vibration is an important concern for encoders, particularly for applications in industrial automation. Well off resonance, the relative movement of the scanner and the bar code will be the same as of the machine parts holding them. If this motion is slow compared to the speed of the scanning spot, the position will be continuously tracked, as in the staring decoder. However, rapid movement will distort the scan line, and may lead to incorrect bit recognition. Since the digital circuit operates by comparing the relative duration of the dark and bright module in each code bit, we can calculate the level of vibration that will cause errors in this comparison by considering the variation in time taken to scan each module.

We take $x=0$ and $\mathrm{t}=0$ as the position and time where the spot begins to scan a particular bar-space pair, for the case of a one module bar and a two module space. We can approximate the undisturbed scan speed across this pair as a constant $v_{s}$, to which is added a vibrational displacement having amplitude, frequency and phase $\mathrm{A}_{v}, \omega_{v}$, and $\phi_{v}$ respectively. Then the spot position is given by

$$
x(\mathrm{t})=v_{s} \mathrm{t}+\mathrm{A}_{v} \cos \left(\omega_{v} \mathrm{t}+\phi_{v}\right)-\mathrm{A}_{v} \cos \left(\phi_{v}\right)
$$

where the final term is added to satisfy $x(0)=0$. The module width is $\mathrm{w}$, and the bit will be misinterpreted if the time $t_{1}$ taken to scan the first module becomes equal to the total time $\left(t_{3}-t_{1}\right)$ taken to scan the following two modules. Then we have

$$
\begin{aligned}
\mathrm{w} & =v_{s} \mathrm{t}_{1}+\mathrm{A}_{v} \cos \left(\omega_{v} \mathrm{t}_{1}+\phi_{v}\right)-\mathrm{A}_{v} \cos \left(\phi_{v}\right) \\
3 \mathrm{w} & =2 v_{s} \mathrm{t}_{1}+\mathrm{A}_{v} \cos \left(2 \omega_{v} \mathrm{t}_{1}+\phi_{v}\right)-\mathrm{A}_{v} \cos \left(\phi_{v}\right) .
\end{aligned}
$$

The time taken to scan one module in the absence of vibration $\left(\mathrm{w} / v_{s}\right)$ is the inverse of the module rate $\mathrm{B}$. If we define $\alpha_{v}=$ $\mathrm{A}_{v} / \mathrm{w}$, then

$$
\begin{aligned}
& 1=\mathrm{Bt}_{1}+\alpha_{v} \cos \left(\omega_{v} \mathrm{t}_{1}+\phi_{v}\right)-\alpha_{v} \cos \left(\phi_{v}\right) \\
& 3=2 \mathrm{Bt}_{1}+\alpha_{v} \cos \left(2 \omega_{v} \mathrm{t}_{1}+\phi_{v}\right)-\alpha_{v} \cos \left(\phi_{v}\right) .
\end{aligned}
$$

The vibrational amplitude at which these equations are satisfied depends on $\phi_{v}$, the worst case (highest sensitivity) being the value of $\phi_{v}$ which gives the minimum $\alpha_{v}$. By a symmetry argument, we can conclude that this worst case will distort the scan times for the bar and space equally, such that $\mathrm{Bt}_{1}=1.5$ and $\mathrm{Bt}_{2}=3$. Then from (12), we can show that the worst case phase $\phi_{v}=\pi-3 \pi \mathrm{f}_{v} / \mathrm{B}$, and noting that $\omega_{v} \mathrm{t}_{1}=3 \pi \mathrm{f}_{v} / \mathrm{B},(11)$ simplifies to give the maximum allowable vibration amplitude

$$
\frac{\mathrm{A}_{v}}{\mathrm{w}}=\frac{0.5}{1-\cos \left(3 \pi f_{v} / \mathrm{B}\right)} .
$$

This is plotted in Fig. 6. The worst case is for $\omega_{v} t_{1}=\pi$, or $\mathrm{f}_{v}=\mathrm{B} / 3$, at which the vibrational amplitude cannot exceed $1 / 4$ of the module spacing. However, this is a relatively high frequency ( $\mathrm{B}=36 \mathrm{kHz}$ in our previous example); the sensitivity at low frequencies is much less.

Another consideration is that an excessive vibration amplitude may reverse the scan direction, which could cause bar-space boundaries to be crossed more than once, and thus give decoding errors. By taking the time derivative of (8) we find that the direction is maintained as long as $\mathrm{A}_{v} \omega_{v}<v_{s}$, or equivalently

$$
\frac{\mathrm{A}_{v}}{\mathrm{~W}}<\frac{1}{2 \pi \mathrm{f}_{v} / \mathrm{B}}
$$

This limit is also shown in Fig. 6, and is the more demanding one for vibration frequencies below $0.07 \mathrm{~B}$ or above $0.45 \mathrm{~B}$, although for high frequencies, filtering of the signal will reduce sensitivity to this effect. For low frequency vibration at amplitudes below these limits, the encoder is effectively able to track the position continuously, with one position update per scan line.

Additional considerations arrive if the external vibration is at a mechanical resonance frequency of the scanner. Then, by 


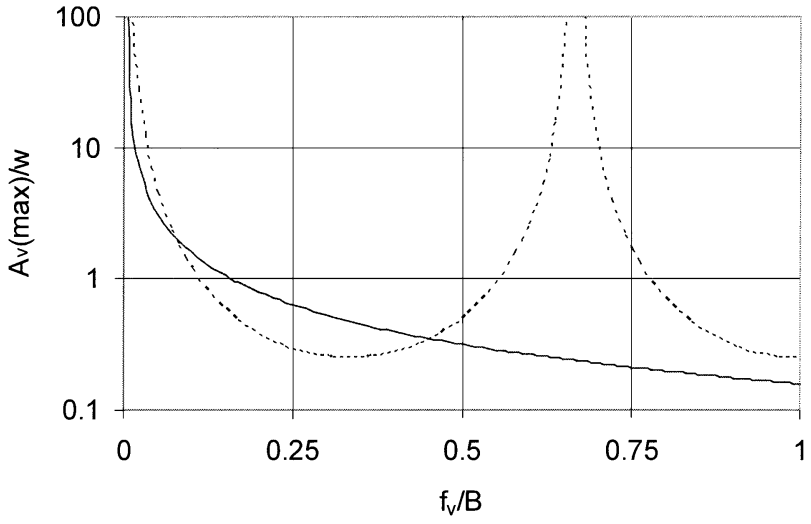

Fig. 6. Maximum allowable vibration amplitude versus vibration frequency; dashed and solid lines correspond to (13) and (14), respectively

analogy with a damped simple harmonic oscillator, the scanner tip displacement will be approximately the vibration amplitude of the scanner module times the $\mathrm{Q}$ of the resonance. Detailed analysis of vibrating cantilever beams is found in Thomson [19] and has been extended for micromachined cantilevers by Syms [20]. Experimental results in [20] indicate Q values for the first resonant mode from about 20 to 100 , for corresponding frequencies from 1000 to $100 \mathrm{~Hz}$. In general, for a vibrating beam, the second mode frequency is about $6 \times$ higher than the first. The scanner will almost certainly be driven at the first resonance, and the higher resonances, for a micromachined beam, will generally be at frequencies above those where machine vibration is appreciable or propagates effectively. The main concern of resonant vibration will then be at or near the drive frequency itself. Here, the effect will mainly be to alter the precise amplitude and phase of the scan. Changes in scan phase and amplitude may also result from alteration of scanner resonant frequency or $\mathrm{Q}$, as a result of changes in ambient temperature or air pressure. The effect of excess scan amplitude will mainly be to reduce the useful scan time available for decoding, lessening the SNR, while scan amplitude reduction will cause complete failure if the number of bits scanned falls below $n$. Otherwise, amplitude changes should not cause position errors directly if the scan remains symmetrical, so that the middle of the scan can be clearly identified. More problematic will be a change in phase between the scan line and drive signal. For this reason, the scan phase should not be determined using the drive signal, but using an additional (end-point) detector, or blanking of the scan line edges. Closed-loop control to keep the scan tracking the resonance is also possible, but adds complexity to the electronics.

\section{EXPERIMENTS}

A first implementation of the proposed technique was carried out using a hybrid integrated fiber optic resonant scanner, as described in [16], and illustrated schematically in Fig. 7. Unmodulated light at $633 \mathrm{~nm}$ is coupled into a single mode fiber with a mode radius of $3.3 \mu \mathrm{m}$. The fiber is mounted on a piezoelectric element, which is driven at the lowest mechanical resonance frequency of the free end of the fiber, which in this case was $233 \mathrm{~Hz}$. The fiber end extends $20 \mathrm{~mm}$ beyond the drive element, and has a maximum peak-to-peak displacement of $\approx 0.5 \mathrm{~mm}$.

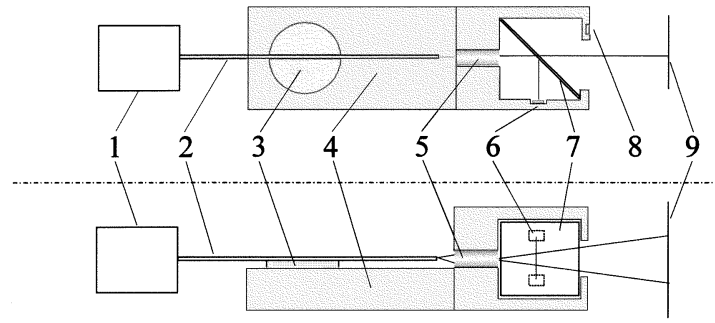

Fig. 7. First prototype scanned encoder: (1) external laser; (2) single mode fiber; (3) piezoelectric disk and cladding mode detector; (4) machined housing; (5) cylindrical GRIN lens; (6) end-point detection photodiode; (7) beam splitter; (8) alternate (reference) detector; (9) scanned code.

This moving fiber tip is imaged onto the bar code using a graded index (GRIN) lens of diameter $2 \mathrm{~mm}, \mathrm{NA}=0.37$, and effective focal length $2.6 \mathrm{~mm}$. With a lens to target distance of $20 \mathrm{~mm}$, and the lens to fiber distance adjusted to optimize focusing, the spot size at the target distance was measured using a moving knife edge, giving a standard deviation $\sigma_{x}$ of $33 \mu \mathrm{m}$.

Previously, use of such a scanner by collecting the return signal in the fiber cladding modes was reported [16]. In this case, detection was also carried out via a separately mounted Siemens BPW34 Si photodiode, connected as the input to a transimpedance amplifier. Additional photodiodes were used in conjunction with a beam splitter mounted between the lens and the target, in order to determine the scan direction, as the reconstruction is based on the forward scan only. Typical SNR was $15 \mathrm{~dB}$, limited by ambient light collected by the optical system. In this case, no optical or high pass electrical filtering was used; the scanner was operated in normal (indoor) daylight conditions, with some shielding of light around the optical system. The SNR was reduced by about $3 \mathrm{~dB}$ at the edges of the scan.

Following the front-end amplifier, the signal is further amplified, differentiated, amplified again, and converted to a digital output using a dual Schmitt trigger stage, as indicated in Fig. 8. The analogue Schmitt trigger acts as a thresholding device, and the digital Schmitt trigger acts as a buffer to the digital circuit. The digital circuit was implemented on an Altera MAX 700084 pin programmable logic chip, for ease of prototyping. The block diagram is given in Fig. 9. The logic level from the Schmitt trigger is effectively polled by the digital circuit at the clock rate $(1 \mathrm{MHz})$. The "width" block measures the number of clock periods between rising and falling edges, while the block "Location" counts the position in the sequence of the bar currently being measured, and provides a threshold width for distinguishing between 1 and 0 values, this being dependent on position in the code because of the sinusoidal variation of the scan speed. The module "delay" provides a virtual mask to blank out the backward scan line, as well as the edges of the forward scan where the scan speed causes the highest degree of distortion. To convert the detected sequence into the index value giving the position of the sequence in the code (i.e., the physical position), a look-up table could be used. However, it was straightforward, and requires less hardware resources, to generate the complete PRBS continuously at the clock speed, in synchronism with a counter giving the equivalent index values. Then the detected sequence can be compared to the PRBS generator, and the counter value transferred to the output 


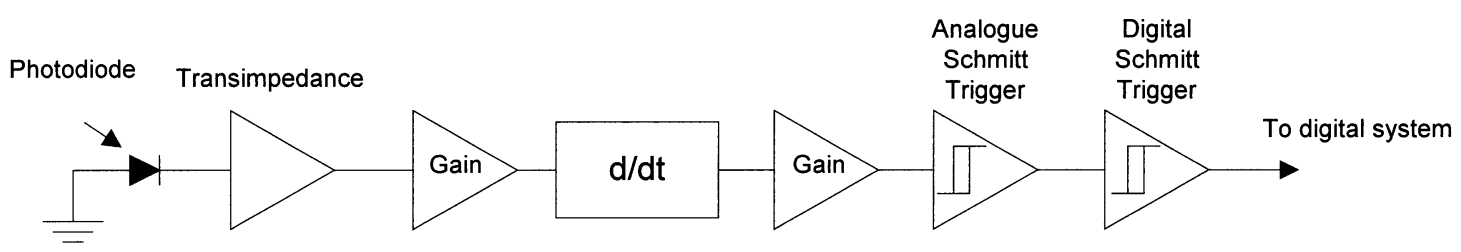

Fig. 8. Block diagram of analogue circuit.

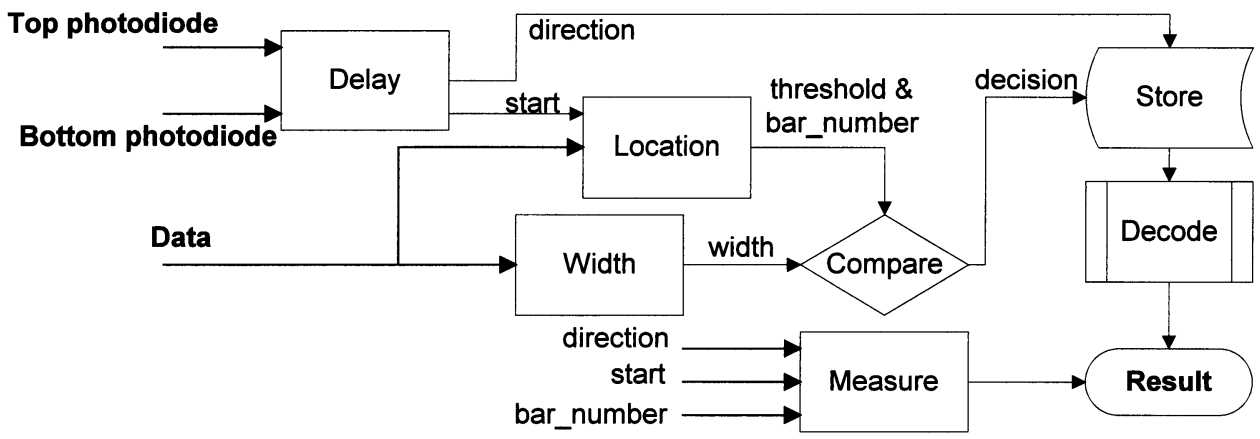

Fig. 9. Block diagram of digital circuit.

when a match is found. This is carried out in the "decode" module.

The system was tested using an $n=6$ code, written on standard paper using a 600-dpi laser printer. The segment width used was $\mathrm{w}=180 \mu \mathrm{m}$. This code could be reliably read to obtain the position at any of the 64 sequence locations. In order to get a better idea of the attainable resolution, the scanner was repeatedly translated across the code line and the positions at which the output value changed were measured by a micrometer and recorded. The average variation in transition location was found to be $\approx 20 \mu \mathrm{m}$. Note that with a spot standard deviation $\sigma_{x}=33 \mu \mathrm{m}$ as measured above, the spot diameter (taken as $4 \sigma_{x}$ ) is $132 \mu \mathrm{m}$, and Fig. 5 indicates that for $\mathrm{w}$ values greater than $2 \sigma_{x}$, the penalty due to blurring is negligible. If 6 - $\mathrm{dB}$ penalty can be tolerated, then we can have $\mathrm{w}=\sigma_{x} / 2.5$, which in this case would be $\approx 13 \mu \mathrm{m}$, leading to a resolution of $3 \mathrm{w} \cong 40 \mu \mathrm{m}$. To obtain further improvement, a smaller scan spot will be required, which can be done straightforwardly by reducing the magnification.

\section{DISCUSSION}

Low cost and simplicity are key advantages for optical encoders, so these will be important criteria for the commercial feasibility of scanned optical encoders. As stated above, integrated optics and micromachining (MOEMS) provide platforms for a high level of integration in such systems, giving reduced size as well as the potential for low cost in high volume markets. This has already been explored for staring position encoders by Sawada et al. [21]. In this device, the laser diode and photodiodes are bonded onto silicon chips on which are deposited fluorinated polyimide optical waveguides, which perform functions including interconnect and phase shifting. A possible MOEMS implementation of the scanned encoder is shown in Fig. 10. A thermal shape bimorph as described in [20] is used as the actuator, with waveguides bringing the light from the optoelectronic chips to the scanning cantilever beam. A directional coupler sep-
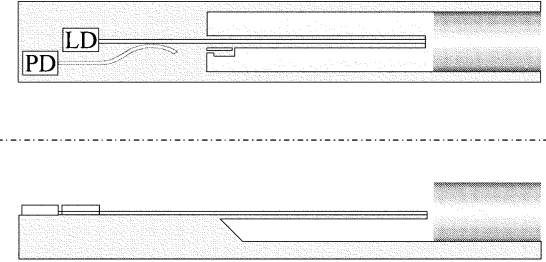

Fig. 10. Proposed silicon integrated scanned encoder, showing laser diode (LD) and photodiode (PD), cantilevered scan arm with thermal actuation structure, GRIN lens housing, waveguides, and directional coupler.

arates transmitted and received light. To reduce the signal loss inherent in this function ( $3 \mathrm{~dB}$ each way), a polarization-dependent coupler could be used, taking advantage of the randomized polarization of the reflected light so that significant loss is only caused on the reflected path. The surface-mounted detector could be replaced by a monolithically integrated one, but such a detector would normally have significantly reduced responsivity, with a corresponding performance reduction.

Another advantage of the scanned encoder over the staring system is its potential use as a remote position sensor for harsh environments, where electronics cannot be adjacent to the moving parts. Both Schmid et al. [22] and Rice et al. [23] have described remote optical staring encoders. In these systems, separate fibers are brought to the detection location for each code bit; high-speed pulsed electronics are then used to multiplex the signals onto a single detection system. The scanned fiber system of Fig. 7 could be used by simply extending the length of the single fiber, keeping the detection and transmission subsystems at the remote end, and thus only the actuator element, which is relatively impervious to electrical noise, would need to be at the measurement end.

\section{CONCLUSION}

The use of scanning for optical position detection is proposed. As a first prototype, an absolute noncontact position 
sensor has been implemented using optical scanning of pseudorandom binary sequences, printed in bar-code format. This technique is more flexible than conventional diode array optical code readers, as the scan range, length and effective sampling rate can be adjusted electronically. It allows the use of signal processing in the time domain, and consequently the recovery of data from low signal-to-noise ratios, and, therefore, should allow increased reader-code separation. It is well suited to implementation using MOEMS, with the whole system apart from the printed code line being potentially realisable as a single chip. This will give high miniaturisation, and potentially low cost and high reliability. There may also be advantages for use in electrically hostile environments.

\section{ACKNOWLEDGMENT}

The authors would like to thank C. Lo for assistance with the design and construction of the prototype system, and A. Holmes and R. Syms for useful discussions and suggestions.

\section{REFERENCES}

[1] M. Dimmler and C. Dayer, "Optical encoder for small drives," IEEE/ASME Trans. Mech., vol. 1, pp. 278-283, 1996.

[2] M. C. Wu, "Micromachining for optical and optoelectronic systems," Proc. IEEE, vol. 85, pp. 1833-1856, 1997.

[3] R. R. A. Syms, "Operation of surface tension self-assembled 3-D microopto-mechanical torsion mirrior scanner," Electron. Lett., vol. 35, pp. 1157-1158, 1999.

[4] M. Ikeda, H. Totani, A. Akiba, H. Goto, M. Matsumoto, and T. Yada, "PZT thin film actuator driven micro-optical scanning sensor by 3-D integration of optocal and mechanical devices," in Proc. 12th IEEE Conf. MEMS, 1999, pp. 435-440.

[5] K. Engelhardt and P. Seitz, "Absolute, high-resolution optical position encoder," Appl. Opt., vol. 35, pp. 201-208, 1996.

[6] M. Durana, R. Gallay, P. H. Robert, and F.-C. Pruvot, "Novel type submicrometer resolution pseudorandom position optical encoder," Electron. Lett., vol. 29, pp. 1792-1794, 1993.

[7] I. J. Good, "Normal recurring decimals," J. London Math. Soc., vol. 21, pp. 167-172, 1946.

[8] J. A. Bondy and U. S. R. Murty, Graph Theory with Applications. London, U.K.: Macmillan, 1976, pp. 181-182.

[9] J. F. Wakerly, Digital Design, Principles and Practices, 3rd ed. London, U.K.: Prentice Hall, 2000, pp. 730-731.

[10] R. D. Elms, "Position Determining Method and Apparatus," U.S. Patent no. $4009377,1977$.

[11] E. M. Petriu, "Scanning method for absolute pseudorandom position encoders," Electron. Lett., vol. 24, pp. 1236-1237, 1988.

[12] T. Pavlidis, J. Swartz, and Y. P. Wang, "Fundamentals of bar code information theory," IEEE Computer, pp. 74-86, Apr. 1990.

[13] F. R. Gfeller and U. Pabst, "Wireless in-house data communication via diffuese infrared radiation," Proc. IEEE, vol. 67, pp. 1474-1486, 1979.

[14] A. J. C. Moreira, R. T. Valadas, and A. M. de Oliveira Duarte, "Optical interference produced by artificial light," Wireless Networks, vol. 3, pp. 131-140, 1997.
[15] S. J. Shellhammer, D. P. Goren, and T. Pavlidis, "Novel signal processing techniques in barcode scanning," IEEE Robot. Automat. Mag., pp. 57-65, Mar. 1999.

[16] D. A. Roberts, R. R. A. Syms, A. S. Holmes, and E. M. Yeatman, "Dual aperture confocal operation of moving fiber bar code reader," Electron. Lett., vol. 35, pp. 1656-1657, 1999.

[17] G. P. Agrawal, Fiber-Optic Communication Systems. New York: Wiley, 1992.

[18] W. Turin and R. A. Boie, "Bar code recovery via the EM algorithm," IEEE Trans. Signal Proc., vol. 46, pp. 354-363, 1998.

[19] W. T. Thomson, Theory of Vibrations with Applications, 4th ed. Cheltenham, U.K.: Nelson Thornes, 2001.

[20] R. R. A. Syms, "Long-travel electrothermally driven resonant cantilever microactuators," J. Micromechan. Microeng., vol. 12, pp. 211-218, 2002.

[21] R. Sawada, E. Higurashi, O. Ohguchi, and Y. Jin, "Long-life micro-laser encoder," Proc. IEEE 13th Int. Conf. MEMS, pp. 491-495, 2000.

[22] E. Schmid, T. Oka, M. Shikai, H. Nakajima, K. Takashima, and T. Usami, "All optical encoder using optical delay," in Proc. 34th SICE Апnи. Conf., 1995, pp. 1187-11901.

[23] T. J. Rice, B. A. Childers, J. W. Borinski, R. L. Crotts, R. W. Harman, and C. D. Boyd, "Passive fiber optic position sensor with digital encoding," in Proc. IEEE Systems Readiness Conf., 2001, pp. 137-147.

Eric M. Yeatman (M'00) received the B.Eng. degree in engineering physics from Dalhousie University, Halifax, NS, Canada, in 1985 and the Ph.D. from Imperial College, London, U.K., in 1989.

Since 1989, he has been a Member of the Staff of the Optical and Semiconductor Devices Group, Electrical and Electronic Engineering Department, Imperial College, currently as a Reader in microengineering and Deputy Head of the Group. His current research includes micromechanical actuators and generators, microstructures for microwave and optical applications, and integrated optical amplifiers.

Paul J. Kushner received the M.Eng. (first class honors) degree in electrical and electronic engineering with management from Imperial College London, U.K., in 2000.

He sat on the IEE London Younger Members Committee. Then he joined Pi Research in Cambridge, U.K., where he is currently designing the digital system for a new control and acquisition product to be used in Formula 1 Motorsport.

David A. Roberts received the B.Sc. degree in physics with electronics from the University of Kent, Canterbury, U.K., in 1983, the Ph.D. degree from the University of Sheffield, Sheffield, U.K., in 1989, and the M.B.A. degree in 1996 from Cranfield University, Cranfield, U.K.

Between 1989 and 1996, he was with the Eastman Kodak Company, Rochester, NY, working on digital imaging and optical data storage technologies. Since joining the Department of Electronic and Electrical Engineering, Imperial College, London, U.K., his research interests have been in the area of MEMS and laser scanning devices. His current interests are in vision science and optometry. 